\title{
Reliability of Mobile 3D Scanning Technologies for the Customization of Respiratory Face Masks
}

\author{
Jonathan BORDUAS ${ }^{1}$, Aude CASTONGUAY-HENRI, Patrick LAURIN, \\ Sean-Philippe VIENS, Daniel BÉLAND \\ Technologies Shapeshift 3D Inc., Montréal (QC), Canada
}

https://doi.org/10.15221/20.34

\begin{abstract}
This paper compares the reliability of four mobile 3D scanning technologies and provides insight and recommendations as to which are of sufficient reliability for the customization of respiratory face mask. More specifically, we will compare the reliability of ARCore: Augmented Faces SDK by Google, the ARKit: Face Tracking SDK by Apple, the ScandyPro app using the raw information of the TrueDepth Structured Light sensor by Apple, and the 3DSizeMe app using the Structure Sensor by Occipital. ARKit and ARCore only provide a $3 \mathrm{~d}$ model of the face, while providing no information of the head shape, we will compare the reliability of extrapolating the head shape from the face scan using Flame Al. ScandyPro and 3DSizeMe do not provide landmarks of the head, as such landmarks for measurements are found using a Deep-MVLM. A subset of anthropometric measurements as suggested by the standard ISO 16976-2:2015 part 2 will be used to assess the reliability of each method and device. We express the reliability of the process in terms of Standard Error of Measurement (SEM).
\end{abstract}

Context: The COVID-19 pandemic has created situations where healthcare providers must wear offthe-shelf N95 masks for long and uninterrupted periods of time. Without applying excessive pressure to the face, it is often difficult to achieve the required airtight seal for the respiratory mask to be effective. Inflammation, pain, and discomfort caused by N95 masks are now the daily reality of numerous healthcare workers. This problem has ignited efforts around the globe to develop custom-fitted respiratory face mask based on the 3D scan of the face.

Results: Reliabilities of different heads scanning technologies have been compared over various measurements. Preliminary results suggest that ARCore, ARKit and 3DSizeMe have sufficient reliability, but are lacking intermethods consistency.

Keywords: Digital anthropometry, 3D reconstruction, 3D scanning, cloud computing, API, measurements, accuracy, reliability, compatibility, MAD, SEM, Bias, MAE, ICC, 3D Face Scan, 3D head scan, custom fit, Al, Artificial Intelligence, Machine Learning, anthropometric measurements, mobile devices, COVID-19, Respiratory Mask.

\section{Introduction}

Respiratory face masks come in different standard sizes. The standard ISO 16976-2:2015 part 2 suggests the use of the ISO digital headform models [1], which come in 5 different sizes. Traditionally, each healthcare professional would try different sizes and undergo a qualitative or quantitative fit test to ensure that there is no leakage into their respirator [2]. A dire supply of mask caused by the current context of a global pandemic [3] is worsened furthermore by a market dominated by single use masks to reduce the chance of cross contamination [4]. Letting healthcare professionals try multiple mask sizes before finding the best fit is not practical. Moreover, the 5 different sizes can only provide a best match of the facial feature of the user. In practice, this leads to healthcare professionals using ill-fitted masks causing excessive pressure and discomfort, especially over extended usage [5].

3D printed, custom fit, respiratory masks remove the need to try multiple sizes and reduce the pressure applied on the face to achieve the necessary airtight seal [6]. Moreover, as the recent pandemic showed [7], 3D printing is a mature and agile method of manufacturing that can be repurposed overnight. Sterilizable hundreds of times [8], SLS 3D printed nylon masks can not only reduce the respiratory masks shortage by being reusable, but also increases the quality of life of the wearer by reducing discomfort and improving safety [6].

1 jonathan.borduas@shapeshift3D.com; shapeshift3D.com 
Initially meant for biometric authentication, augmented reality and depth effects in photographs, handheld 3D scanners integrated with the depth sensing hardware are now a common occurrence in billions of mobile phones. iPhones X, XR, XS, 11, 11 Pro now feature a frontal 3D scanner [9] while the iPad Pro 2020 features a back facing 3D scanner [10]. The sales of iPhones featuring a frontal 3D scanner now represents $54 \%$ of the North American mobile phone market [11], [12] and $25 \%$ of the global market [13]. Other flagships such as the Samsung Note10+ now encompass similar technology [14]. The innovation should continue both in terms of brand adoption as well as in democratization. Phone apps such as ScandyPro can utilize such depth sensing capabilities.

Specialized hardware modules can also be attached to mobile phones and tablet, such as the Structure Sensor by Occipital [15]. Mobile apps, for example 3DSizeMe, can connect to these modules and guide the user through the scanning various parts of the body, including the head [16].

In addition, Al technologies, such as the ARCore Augmented Faces SDK, allow to convert a camera phone into a 3D scanner for specific applications, without the need for depth sensing hardware [17]. The outputted 3D scan is scaled properly using the mobile phone IMU [18]. The generated scans always have the same mesh topology and can thus be used to position landmarks [19]. This technology, both accessible on iOS and Android phones can be used on more than $17 \%$ of the current devices on the market [20].

In the case of the ARKit: Face Tracking SDK by Apple, an Al uses the phone camera and depth sensing capabilities of iPhones [21] with the aim to create a more precise 3D scan than with Al alone, as well as an easier scanning experience than with depth sensing alone.

The orthotics and prosthetics (O\&P) industry keeps making increased use of low-cost handheld 3D scanner [22]. For example, already $41 \%$ of Australia's podiatrists use 3D scanning to capture the patient's foot shape and create custom made foot insoles [23]. These professionals undergo trainings with the scanning hardware to provide better 3D scans. In the context of self-scanning or consumer scanning, such training sessions are impractical. Even in organizations with training programs, where operator are healthcare professionals, scans regularly exhibit flaws including, but not limited to, voids and missing area, stitching problems when registering scan patches, and outliers. It has been observed that most users are now capable of properly evaluating scan quality [24].

As the 3D scanning process, like most handheld measurement tools, is affected by both the tool reliability and the operator's skills, we must not study the reliability of the tool itself, but of the operator and of the tool as a whole. Therefore, imprecise but easy to use tools might be more reliable than accurate but complex to operate tools [25].

In this light, we study the reliability of different scanning technologies: making sure that our design of experiments will encompass all errors that may be caused by the operator itself.

This paper presents a study to quantify the reliability of the various 3D scanning technologies in the context of custom respiratory face mask.

\section{Methods}

\subsection{Design of experiment}

Three females and six males were scanned three times for each of the four scanning technologies compared in this study for a total of 108 scans. The subjects were self-scanning with ARKit, ARCore and ScandyPro as these scanners are front-facing and meant to be operated by the user directly. The 3DSizeMe app requires being scanned by a trained professional as this scanner is rear facing and meant to be operated by another person.

We measure reliability of the four scanning technologies over 8 measurements, a subset of anthropometric measurements used by the standard ISO 16976-2:2015 part 2. This standard uses Euclidian distances and geodesic distances measured between landmarks located all over the head.

Three scans were generated per subject and methods; the experiment was designed to exceed the minimal sample size and repetition number for significantly testing $\mathrm{H}_{0}: p=0.8$ versus $\mathrm{H}_{1}: p>0.8$ [25]. The ICC was evaluated and compared to that of other reconstruction methods at the $5 \%$ significance level with $80 \%$ power. To better assess the reliability of our method, the one-sided $95 \%$ confidence interval lower bound ICC was determined. 


\subsection{Data processing}

To obtain a given measurement on a given scan, we need a full head scan, and we need the landmarks to be appropriately placed. ARKit and ARCore provide a 3D registered model of the face, while providing no information of the head shape. It is thus easy to identify landmarks over the face, but additional work is required to do so for other sides of the head and for the neck. We hypothesize that an Al model of the head could extrapolate the results from ARKit and ARCore accurately. For that purpose, we use the TF-Flame AI model [26] to extrapolate the head shape based on landmarks extracted from the ARKit and ARCore facial mesh. We then combine the data from either ARKit or ARCore with the full head mesh generated by TF-Flame. In order to do that, we use the mesh fitting capabilities of TF-Flame to output a new final mesh that takes into account the back of the head initially generated by the first TFFlame run, and the face from either ARKit or ARCore. This helps generate forehead and neck areas that are more representative of the subject. Since the TF-Flame Al generates a registered model, it is possible to directly extract the needed measurement landmarks.

ScandyPro and 3DSizeMe provide a full model of the head, but each output meshes are unregistered and require a reconstruction process. We are using Shapeshift 3D Repair algorithms [27] to reconstruct the scan, we then use the Deep-MVLM AI model to locate 73 landmarks [28]. We use the 73 landmarks to find the symmetry plane and mirror the mesh, this helps improve the mesh accuracy by averaging the left and right sides. We then symmetrize the 73 landmarks and use them to register a mesh containing all the necessary measurements landmarks as indicated in ISO 16976-2:2015 part 2.

Figure 1 shows an example of raw scan for each of the four technologies.
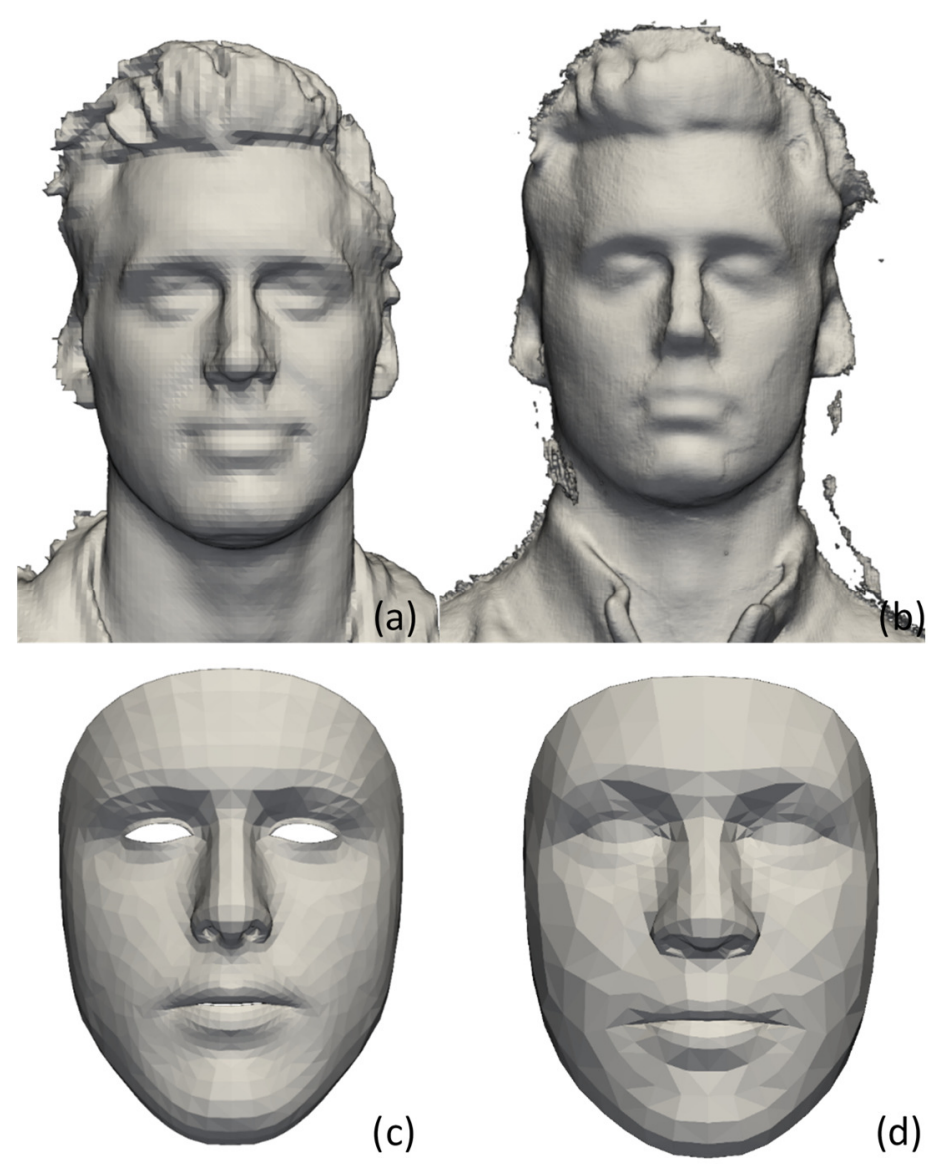

(d)

Figure 1: Raw scan taken using a) ScandyPro; b) 3DSizeMe c) ARKit; d) ARCore

While Deep-MVLM, the scan reconstruction process and Flame Al might introduce additional errors, we consider these steps necessary for the customization of a respiratory face mask, as well as for the measurement of the accuracy of the 3D scanning technologies. Their errors must thus be considered. 


\subsection{Analytic procedures}

In our reliability analysis, we use measurements derived from the anatomical and non-anatomical landmarks indicated by ISO16976-2:2015. Table 1 shows details of each measurement.

Table 1. Anatomical measurements used for reliability analysis.

\begin{tabular}{|c|c|c|}
\hline $\begin{array}{c}\text { ISO16976-2_2015 } \\
\text { Measurement }\end{array}$ & Type of measurement & Associated landmarks \\
\hline Minimum frontal breadth & Straight-line distance & $\begin{array}{l}\text { Right frontotemporale landmark } \\
\text { Left frontotemporale landmark }\end{array}$ \\
\hline Face width & Straight-line distance & $\begin{array}{l}\text { Right Zygion landmark } \\
\text { Left Zygion landmark }\end{array}$ \\
\hline Bigonial Breadth & Straight-line distance & $\begin{array}{l}\text { Right Gonion landmark } \\
\text { Left Gonion landmark }\end{array}$ \\
\hline Menton-Sellion length & $\begin{array}{l}\text { Distance in the } \\
\text { midsagittal plane }\end{array}$ & $\begin{array}{l}\text { Menton landmark } \\
\text { Sellion landmark }\end{array}$ \\
\hline Interpupillary distance & Straight-line distance & $\begin{array}{l}\text { Right pupil landmark } \\
\text { Left pupil landmark }\end{array}$ \\
\hline Head breadth & Straight-line distance & $\begin{array}{l}\text { Right Tragion } \\
\text { Left Tragion }\end{array}$ \\
\hline Nose protrusion & Straight-line distance & $\begin{array}{l}\text { Pronasal landmark } \\
\text { Subnasale landmark }\end{array}$ \\
\hline Nose Breadth & Straight line distance & $\begin{array}{l}\text { Right alare landmark } \\
\text { Left alare landmark }\end{array}$ \\
\hline Nasal root breadth & Straight line distance & $\begin{array}{l}\text { Nasal Root Point Right } \\
\text { Nasal Root Point Left }\end{array}$ \\
\hline Subnasal-sellion length & Straight line distance & $\begin{array}{c}\text { Sellion landmark } \\
\text { subnasale landmark. }\end{array}$ \\
\hline Bitragion chin arc & Surfacic distance & $\begin{array}{c}\text { Right Tragion landmark } \\
\text { Chin landmark } \\
\text { Left Tragion landmark }\end{array}$ \\
\hline Bitragion coronal arc & $\begin{array}{l}\text { Top Surfacic distance } \\
\text { along the coronal plane }\end{array}$ & $\begin{array}{l}\text { Right Tragion landmark } \\
\text { Left Tragion landmark }\end{array}$ \\
\hline Bitragion frontal arc & Surfacic distance & $\begin{array}{l}\text { Right Tragion landmark } \\
\text { Right Frontotemporale } \\
\text { Left Frontotemporale } \\
\text { Left Tragion landmark }\end{array}$ \\
\hline Bitragion subnasale arc & Surfacic distance & $\begin{array}{l}\text { Right Tragion landmark } \\
\text { Subnasal landmark } \\
\text { Left Tragion landmark }\end{array}$ \\
\hline Head circumference & $\begin{array}{l}\text { Double the surface } \\
\text { distance }\end{array}$ & $\begin{array}{l}\text { Right Tragion landmark } \\
\text { Glabella landmark } \\
\text { Left Tragion landmark }\end{array}$ \\
\hline Head length & $\begin{array}{l}\text { Double the distance in } \\
\text { the midsagittal plane }\end{array}$ & $\begin{array}{l}\text { Glabella landmark } \\
\text { Tragion landmark }\end{array}$ \\
\hline Lip length & Straight-line distance & $\begin{array}{l}\text { Right chelion landmark } \\
\text { Left chelion landmark }\end{array}$ \\
\hline Maximum frontal breadth & Straight-line distance & $\begin{array}{l}\text { Right zygofrontale landmark } \\
\text { Left zygofrontale landmark }\end{array}$ \\
\hline Neck circumference & $\begin{array}{l}\text { Circumference } \\
\text { measurement }\end{array}$ & $\begin{array}{l}\text { perpendicular to the next axis at the } \\
\text { height of the Adams apple. }\end{array}$ \\
\hline Stature & Straight-line distance & $\mathrm{N} / \mathrm{A}$ \\
\hline Weight & $\mathrm{N} / \mathrm{A}$ & $\mathrm{N} / \mathrm{A}$ \\
\hline
\end{tabular}


Out of the measurements listed, we selected eight based on whether the associated landmarks could be detected by the Deep-MLVM algorithm, and on their relevance in the context of respiratory masks. The selected measurements are listed below. Table 2 presents the associated landmarks and their definition.

1. Bigonial Breadth

2. Interpupillary distance

3. Nose protrusion

4. Nose Breadth

5. Nasal root breadth

6. Bitragion chin arc

7. Bitragion subnasale arc

8. Lip length

Table 2. Anatomical measurements used for the reliability analysis.

\begin{tabular}{cc}
\hline IS016976-2_2015 landmark & Definition \\
\hline Gonion & Most lateral, inferior and posterior point of the mandible \\
\hline Pupil & Center of each eye \\
\hline Pronasale & Tip of the nose \\
\hline Subnasale & Juncture of the groove of the upper lip with the nose \\
\hline Alare & Furthest point of each wing of the nose \\
\hline Nasal root point & Halfway between the bridge of the nose and the tip of the eye \\
\hline Tragion & Highest juncture between the ear and the head \\
\hline Chin & Most protruding point along the chin \\
\hline Chelion & Tip of the lips \\
\hline
\end{tabular}

Figure 2 shows examples of reconstructed scans performed with each scanning technology and their detected landmarks.
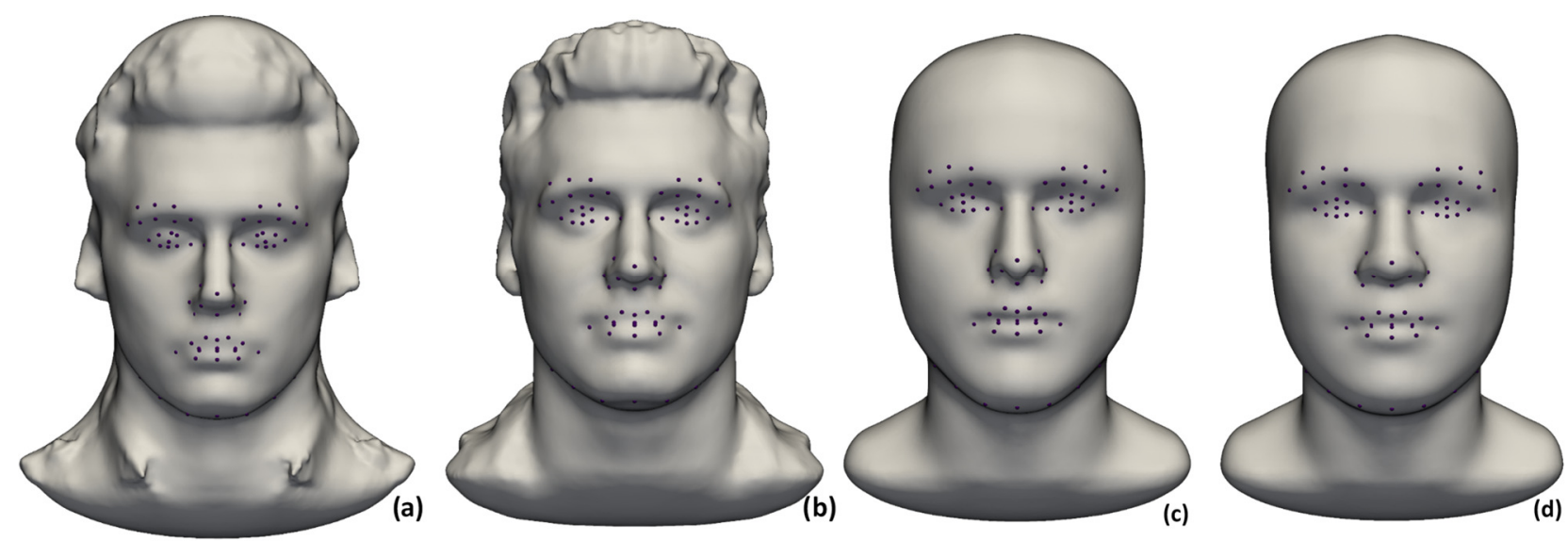

Figure 2: Reconstructed scan with landmarks using a) ScandyPro; b) 3DSizeMe c) ARKit; d) ARCore

Those values are measured for each scan, which allows us to determine the standard error of measurement (SEM), as well as the Intraclass Correlation Coefficient (ICC) of each of the four scanning technologies. 


\section{Result and discussion}

In table 3, we present the SEM for the four 3D scanning technologies of interest, for each of the eight selected measurements.

Table 3. Standard Error of Measurement (SEM) in mm of different 3D scanning technologies.

\begin{tabular}{cccccc}
\hline Measurement & $\begin{array}{c}\text { ScandyPro } \\
{[\mathrm{mm}]}\end{array}$ & $\begin{array}{c}\text { ARKit } \\
{[\mathrm{mm}]}\end{array}$ & $\begin{array}{c}\text { ARCore } \\
{[\mathrm{mm}]}\end{array}$ & $\begin{array}{c}\text { 3DSizeMe } \\
{[\mathrm{mm}]}\end{array}$ & $\begin{array}{c}\text { Intermethod } \\
{[\mathrm{mm}]}\end{array}$ \\
\hline Bigonial Breath & 4.3 & 1.0 & 1.0 & 1.3 & 6.9 \\
\hline Interpupillary distance & 2.5 & 1.5 & 0.5 & 0.4 & 2.8 \\
\hline Nose protrusion & 1.0 & 0.2 & 0.2 & 0.3 & 0.9 \\
\hline Nose breadth & 2.4 & 0.3 & 0.3 & 0.7 & 2.4 \\
\hline Nasal root breadth & 1.1 & 0.2 & 0.2 & 0.5 & 1.4 \\
\hline Bitragion chin arc & 9.0 & 3.5 & 4.0 & 4.3 & 13.2 \\
\hline Bitragion subnasal arc & 8.9 & 6.8 & 5.0 & 4.6 & 11.8 \\
\hline Lip length & 2.4 & 0.6 & 0.3 & 1.1 & 2.5 \\
\hline
\end{tabular}

Table 3 shows high errors with the ScandyPro scanning technology, suggesting either a noisier scan or greater variability between scans. Errors over $3 \mathrm{~mm}$ are obtained with all methods for the bitragion measurements, which are also the measurements with the greater values. Table 4 shows relative errors of all four methods for the bitragion measurements.

Table 4. Relative error of different 3D scanning technologies for bitragion measurements.

\begin{tabular}{ccccc}
\hline Measurement & ScandyPro & ARKit & ARCore & 3DSizeMe \\
\hline Bitragion chin arc & $3.5 \%$ & $1.4 \%$ & $1.6 \%$ & $1.7 \%$ \\
\hline Bitragion subnasal arc & $3.3 \%$ & $2.5 \%$ & $1.9 \%$ & $1.7 \%$ \\
\hline
\end{tabular}

The relative errors again suggest lower accuracy for ScandyPro, while the result of $2 \%$ suggests acceptable error margins for all other technologies.

As for Intraclass Correlation Coefficients (ICC), we calculated an average value of $0.68 \pm 0.10$ for ScandyPro, $0.94 \pm 0.08$ for ARKit, $0.96 \pm 0.05$ for ARCore, and $0.93 \pm 0.04$ for 3DSizeMe. The ICC results for ScandyPro correspond to a moderate reliability and can be explained in part by the difficulty of use. A moderate ICC suggests ScandyPro is poorly suited for face scans, which require great precision to ensure a perfect fit, and therefore a high level of comfort and protection. ICCs for ARCore, ARKit and 3DSizeMe indicate excellent reliability for all three technologies.

Our $p$-values for this study are in average higher than 0.001 . While some measurements reach statistical significance, most indicate additional data is required to confirm reliability. For example, due to the high SEM, all ScandyPro's p-value are higher than 0.9. Some $p$-value however, especially for ARCore, are lower than 0.001 , indicating that the ICC for those measurements are significant. This further suggests for ARCore to be a reliable scanning technology.

These numbers suggest that our study did not use enough scans. Therefore, in general, we cannot say our sample is statistically representative or significant. The $95 \%$ lower bounds of the ICCs are 0.19 for ScandyPro, 0.79 for ARKit, 0.86 for ARCore and 0.77 for 3DSizeMe. Since no lower bound falls into the same reliability rating as its corresponding ICC, those numbers again indicate that more data is required to better evaluate the four technologies.

The intermethods results relate to the reliability of all four methods together. The average ICC of 0.44 shows each technology results in different values for a same measurement. This low consistency between methods shows all technologies require additional calibration. A study with ground truth would need to be conducted to properly evaluate the bias for each method. 


\section{Conclusion and further work}

The aim of this study was to evaluate the reliability of four 3D scanning technologies: ScandyPro, ARKit, ARCore and 3DSizeMe. While we lacked a large enough sample to reach statistical significance, preliminary results suggest ARKit, ARCore and 3DSizeMe to be reliable and therefore suitable for 3D head scans in the context of custom-fit respiratory masks. On the other hand, intermethods' ICCs show a lack of consistency, and therefore suggests additional calibration is required for each method. Further studies will be done to evaluate the bias of each method and develop appropriate calibration. A way to evaluate the bias would be to print 3D head forms and use them as a baseline.

While 3DSizeMe obtains similar results as ARKit and ARCore in terms of reliability, it requires a second person to take the scan, as well as the specific hardware compatible with the app. Therefore, even if they are as reliable, ARKit and ARCore represent simpler and more accessible solutions to 3D face scans.

After additional validation, we believe the ARKit and ARCore 3D scanning technologies will allow users to send reliable and representative scans of their face, enough to generate custom-fit respiratory masks. This approach will be tested at scale with the commercialization of the BeyondFit ${ }^{\mathrm{TM}}$ mask, a Shapeshift 3D-enabled, reusable, 100\% recyclable, custom-fit, respiratory face mask [29].

\section{References}

[1] Centers for Disease Control and Prevention, "NIOSH Anthropometric Data and ISO Digital Headforms," 23 April 2020. [Online]. Available: https://www.cdc.gov/niosh/data/datasets/rd10130-2020-0/default.html.

[2] Government of Canada, "Safety and performance specifications for respirators during COVID19: Guidance for Canadian manufacturers," 25 August 2020. [Online]. Available: https://www.canada.ca/en/health-canada/services/drugs-health-products/covid19industry/medical-devices/personal-protective-equipment/medical-masks-respirators/safetyperformance-specifications.html.

[3] A. Jacobs, M. Richtel and M. Baker, "'At War With No Ammo': Doctors Say Shortage of Protective Gear Is Dire," The New York Times, 19 March 2020. [Online]. Available: https://www.nytimes.com/2020/03/19/health/coronavirus-masks-shortage.html.

[4] Centers for Disease Control and Prevention, "Strategies for Optimizing the Supply of Facemasks," 28 June 2020. [Online]. Available: https://www.cdc.gov/coronavirus/2019ncov/hcp/ppe-strategy/face-masks.html.

[5] A. Gefen and K. Ousey, "Update to device-related pressure ulcers: SECURE prevention. COVID-19, face masks and skin damage," Journal of Wound Care, vol. 29, no. 5, pp. 245-259, 2020.

[6] L. Degueldre and Al, "Improving the Fit of Respiratory Face Masks Through 3D Scanning, Finite Elements Analysis and Additive Manufacturing," in 3DBodyTech, Lugano, Switzerland, 2020.

[7] S. Belhouideg, "Impact of 3D printed medical equipment on the management of the Covid19 pandemic," The International journal of Health Planning and Management, vol. 35, no. 5, pp. 1014-1022, 2020.

[8] M. J. Haerst, R. Wolf, M. Schönberger and E. Wintermantel, "Ageing processes in laser sintered and injection moulded PA12 following hygienic reprocessing," Rapid Prototyping Journal, vol. 21, no. 3, pp. 279-286, 2015.

[9] Cyborg Standard, [Online]. Available: https://www.standardcyborg.com/profile. [Accessed 2019].

[10] "Ipad Pro," Apple, 2020. [Online]. Available: https://www.apple.com/ca/fr/ipad-pro/. [Accessed October 2020].

[11] "Mobile Vendor Market Share North America," Stat Counter Global Stats, [Online]. Available: https://gs.statcounter.com/vendor-market-share/mobile/north-america. [Accessed October 2020]. 
[12] "Apple's iPhone XR was the best Selling Smartphone in the U.S. in Q2 with a Whopping 48\% Market Share," Patently Apple, [Online]. Available: https://www.patentlyapple.com/patentlyapple/2019/07/apples-iphone-xr-was-the-best-selling-smartphone-in-the-us-in-q2-with-awhopping-48-market-share.html. [Accessed 12 October 2020].

[13] "Mobile Vendor Market Share Worldwide," Stat Counter Global Stats, [Online]. Available: https://gs.statcounter.com/vendor-market-share/mobile/worldwide.

[14] "Samsung Galaxy Note 10," Wikipedia, 30 August 2019. [Online].

[15] "Structure," Occipital, [Online]. Available: https://structure.io/.

[16] "3DSizeMe," Techmed3D, [Online]. Available: https://techmed3d.com/products/3dsizeme/.

[17] "Augmented Faces," Google, [Online]. Available:

https://developers.google.com/ar/develop/ios/augmented-faces/overview.

[18] "ARCore: Fundamental concepts," Google, 20 July 2020. [Online]. Available: https://developers.google.com/ar/discover/concepts\#motion_tracking.

[19] "ARCore: Creating custom textures and 3D models for Augmented Faces," Google, 16 June 2020. [Online]. Available: https://developers.google.com/ar/develop/developer-guides/creatingassets-for-augmented-faces.

[20] "ARtillery Intelligence," Ar Insider, [Online]. Available: https://arinsider.co/about/. [Accessed October 2020].

[21] "Face Tracking with ARKit," Apple Inc, [Online]. Available: https://developer.apple.com/videos/play/tech-talks/601/.

[22] M. Hassmann, S. Stoeger, J. Dastl and W. Krach, " Scanning Procedure of Female Torso Using Low-Cost Hand-Held Sense 3D Scanner," in 3DBODY.TECH 2018 - 9th Int. Conference and Exhibition on 3D Body Scanning and Processing Technologie, 2018.

[23] L. S. Chapman and al, "Foot orthoses for people with rheumatoid arthritis: a survey of prescription habits among podiatrists," Journal of Foot and Ankle Research, vol. 12, no. 1, p. 7 , Jan 2019.

[24] X. Yin, B. D. Corner and A. Razdan, "EARS: Toward Fast Analysis of 3D Human Scans," 2010.

[25] M. Eliasziw, S. Young, M. G. Woodbury and K. Fryday-Field, "Statistical Methodology for the Concurrent Assessment of Interrater and Intrarater Reliability: Using Goniometric Measurements as an Example," Physical therapy, vol. 74, pp. 77-88, 1994.

[26] T. Li, T. Bolkartl, M. J. Black, H. Li and J. Romero, "Learning a model of facial shape and expression from 4D scans," ACM Transactions on Graphics, vol. 36, no. 6, pp. 1-17, 2017.

[27] P. Laurin, D. Béland and J. Borduas, "A fully automated and unsupervised cloud API for the reliable reconstruction of ra w3D surface scans data," in 3D BodyTech, Lugano, 2019.

[28] R. R. Paulsen, K. A. Juhl, T. M. Haspand, T. Hansen, M. Ganz and G. Einarsson, "Multi-view consensus CNN for 3D facial landmark placement," in Computer Vision - ACCV 2018, Perth, Australia, 2018.

[29] "The BeyondFit Mask," Technologies Shapeshift 3D, [Online]. Available: https://www.shapeshift3d.com/beyond-fit-mask. 